\title{
Role of CCL21 on the Transcriptional Regulation of MHC II in Malignant Glioma Infiltrating Plasmacytoid Dendritic Cells
}

\author{
Sharlé Newman ${ }^{1}$, Sreenivasulu Chintala², Mario Henriquez ${ }^{2}$, Mahua Dey $^{2}$ \\ ${ }^{1}$ Indiana University School of Medicine, ${ }^{2}$ Indiana University School of Medicine, \\ Department of Neurosurgery
}

Background and Hypothesis: Glioblastoma (GBM) is a malignant brain tumor characterized by high tumor heterogeneity and an immunosuppressive tumor microenvironment (TME). Immunomodulation approaches have been investigated, but outcomes remain poor. Several studies describe the functional deregulation of immune cells including, T cells, dendritic cells (DC), and macrophages. Plasmacytoid dendritic cells (pDC), which accumulate in the GBM TME, are shown to have an immunosuppressive phenotype characterized by a lack of IFN- $\alpha$ secretion and upregulation of MHC II. MHC II presentation is transcriptionally regulated by several factors produced by tumor cells including, TGF $\beta$, TNFa, and IL10 through the modulation of CIITA, the catalytic component of the enhanceosome. GBM tumor cells secrete several chemokines/cytokines, which may regulate $\mathrm{MHC}$ II expression in pDCs. We hypothesize that chemokine CCL21 transcriptionally upregulates MHC II through the activation of CIITA in pDC.

Experimental Design/Project methods: We performed experiments using two GBM tumor cells models GL261 and CT2A and used western blot, PCR, immunohistochemistry, immunofluorescence, and flow cytometry to determine the levels of CCL21 and its ligands ACKR3/4 in tumor cells and pDC.

Results: We observed overexpression of CCL21 in GBM and upregulation of $\mathrm{MHCll}$ in tumor associated pDC. We predict that inhibition of CCL21 will lead to downregulation of $\mathrm{MHC}$ II in tumor associated $\mathrm{pDC}$ which could potentially lead to reversal of the immunosuppressive TME by presenting the antigens to T cells.

Conclusions/Potential Impact: The results of this study can elucidate novel mechanisms of $\mathrm{MHCl}$ regulation and identify CCL21 as a potential therapeutic target for immunotherapy development in GBM. 